\title{
Quality assessment in surgery: where do we stand now and where should we be heading?
}

\author{
S. Weyers • S. Van Calenbergh • Y. Van Nieuwenhove • \\ G. Mestdagh • M. Coppens • J. Bosteels
}

Received: 7 January 2014 / Accepted: 11 March 2014 /Published online: 21 March 2014

(C) Springer-Verlag Berlin Heidelberg 2014

\begin{abstract}
While surgery is gaining in efficiency it is equally getting more and more complex. Meanwhile patients are getting more and more demanding. In the past decades, safety and quality have become prominent criteria by which surgical care is evaluated. Several important factors can be identified which are influencing the quality of surgical care, in our view these factors can be classified into four major groups: the team of caretakers, the patient, the material, and the procedure. For all of these factors, a high level of knowledge and optimal communication is crucial to guarantee a high standard of care and minimize the chance of complications. Different quality assessment tools are currently used in surgery. Databases of surgical procedures have the potential to offer an enormous amount of information on the quality of care. However, the implementation of comprehensive databases is difficult and expensive, while its value is overshadowed by possible underreporting. Introducing surgical checklists is a cheap yet efficient way to increase both the safety and the quality of
\end{abstract}

\section{S. Weyers $(\triangle)$}

Department of Obstetrics and Gynecology,

Ghent University Hospital, Ghent, Belgium

e-mail: steven.weyers@ugent.be

S. Van Calenbergh

Department of Obstetrics and Gynecology, AZ Turnhout,

Turnhout, Belgium

Y. Van Nieuwenhove

Department of Surgery, Ghent University Hospital, Gent, Belgium

G. Mestdagh

Department of Obstetrics and Gynecology, Ziekenhuis

Oost-Limburg (ZOL), Genk, Belgium

\section{Coppens}

Department of Anaesthesiology, Ghent University Hospital, Gent, Belgium

\section{J. Bosteels}

Department of Obstetrics and Gynecology, Imelda Hospital,

Bonheiden, Belgium surgical care. Nevertheless, its implementation is sometimes opposed since they slow down the patient flow. The risk of complications tends to increase when a new technique is introduced. Therefore, quality assurance (QA) programs have to be implemented. Surgical simulation training is rapidly becoming a necessary adjunct to traditional patient-based training models. Finally, key performance indicators (KPI) can be used for measuring the success of medical interventions such as surgery. For the near future, the introduction of one comprehensive medical file per patient could be a major step in increasing the safety and efficiency of our medical deeds. In parallel, a nationwide prospective registry for surgical interventions should be introduced. Postgraduate surgical training should be organized by the national professional groups and should be adapted to the local needs. A system of accreditation for specific interventions should be introduced guaranteeing their state-of-the-art application.

Keywords Quality indicators · Surgical procedures · Education

\section{Background}

Safety and quality have become prominent criteria by which surgical care is evaluated. Up till now, due to a tremendous variance in morbidity figures, the interpretation and comparison of surgical results is very difficult. This is mainly caused by the absence of international guidelines on complications: when talking about complications we need to talk the same language, but at the present, no universally accepted classification of surgical complications exists. On the other hand, we have to admit that differences in case mix and observation time might play an important role.

What seems so hard for us to accomplish in surgery has become common practice in private industry for more than six 
decades: the Japanese car builder Toyota made the pioneering effort to improve quality as early as in the 1950s [1].

In surgery, there are in fact four major determinants of the quality of care: the team of caretakers (physicians, both in hospital and in community, nurses, logistical staff, etc.), patient (including its comorbidity and pathology), material (surgical material, medication, etc.), and procedure.

We aim to address the impact of each of these four factors in the present paper. Moreover, we aim to present an overview of the quality assessment tools that are currently used in surgical practice. Where appropriate, we will try to suggest future applications.

\section{Major determinants of the quality of care in surgery}

\section{Team of caretakers}

A first prerogative for optimal care consists of a good basic training of all the caretakers involved. Such training should ideally be lifelong; surgeons as well as nurses and logistic personnel should be offered appropriate courses to optimize the patient care in a continuously changing environment. Patient care is becoming more and more demanding and stressful, and as a consequence, the risk of complications gradually increases [2].

Moreover, the principle of medical team training (MTT) has proven to be an important tool to improve surgical efficiency. This consists of a thorough (month-long) planning with all the facilities surgical teams, followed by an on-site learning session with each individual team. MTT is able to improve teamwork, to enhance safety attitudes, and to reduce errors [3]. Moreover, Neily et al. [4] managed to demonstrate that the introduction of such a training was associated with a reduction in surgical mortality rate.

Indeed, optimal communication between different caretakers is a second important factor in patient care: all relevant information on the patient's unique situation should be clearly and fully communicated not only among the treating physicians [organ specialists, surgeons, general practitioners (GPs), anesthesiologists, etc.] but also equally important between surgeons and the other caretakers (nurses, pharmacy, logistics, etc.) in the hospital and between hospital caretakers and those taking care of the patient before and after hospital discharge. The optimization of the patient's condition for surgery should start in primary care: general practitioners can play a major role by identifying potential risks of morbidity (anemia, obesity, suboptimal diabetic control, reduced renal function, etc.). Preoperative correction of even minor anemia can significantly reduce the postoperative need for transfusion (http://www. transfusionguidelines.org.uk). A unique nationwide medical file per patient, accessible by all treating caretakers, would undoubtedly improve communication and transmission of important information between caretakers.
During any intervention, the whole team involved should ensure the best possible treatment not only on the technical point of the intervention itself but also on the level of fluid management (preventing dehydration or overfilling), patient temperature (preventing hypothermia), patient positioning, etc. Surgeons have to ensure that the procedure is as minimally invasive as possible, however, not at the cost of increasing the risks for the patient. All perioperative actions (usage of surgical drains, nasogastric tubes, bladder catheters, analgesic treatment, etc.) should be questioned on their proven benefit and only be used if there is clinical evidence to support their effectiveness [5]. After surgery patients should receive the best possible rehabilitation (early and late recovery) including timely resumption of drinking and eating, addition of nutritional supplements if necessary, early mobilization if possible, adequate analgesia, early removal of drains and catheters, etc.

After discharge, a seamless and continuing care has to be pursued; therefore, all necessary information should be communicated to the patient's family, GP, and other caretakers outside the hospital. Written information on the necessary aftercare (wound care, allowed analgesia, mobilization, etc.), possible complications, and warning signs should be given; moreover, a (temporary) letter of dismissal should be accompanying the patient upon his departure.

\section{Patients}

The benefit of preoperative assessment of the surgical patient has been recognized since long and aims at reducing over-all perioperative risks [6]. It is essential to consider patient related factors, social related factors, and the duration and extent of the surgery. Routine gynecological surgery is classified as "low"-risk surgery (cardiac risk $<1 \%$ ). Major oncologic surgery is classified as "intermediate" risk (cardiac risk 1-5\%). "High"-risk procedures (cardiac risk $>5 \%$ ) are aortic surgery and major vascular or peripheral vascular surgery.

Specific preoperative assessment services, performed by trained and competent assessors who can order and/or perform investigations and make referrals if necessary, are fundamental to identifying preoperative risk [7]. Patients are screened with paper or electronically based questionnaires depending on the American Society of Anesthesiologists (ASA) score. Such preoperative assessment services should include direct access to the treating anesthesiologist, surgeon, and other healthcare professionals who are competent to review the findings and agree on management strategies which are to optimize the patient's condition before surgery.

Preoperative exams ideally can be performed 2 to 3 weeks before surgery, thus giving enough time to order additional tests if necessary, while being close enough to the admission date to have up-to-date information. Thus, day-before-surgery admissions can be avoided and nearly all patients can be admitted on the day-of-surgery. Nurses specifically trained 
to assist in the planning of the surgical procedure can greatly facilitate this process: planning of the necessary preoperative exams, run through the different steps of the procedure indicating possible complications, referring the patient to the hospital social service if assistance upon discharge is necessary, planning of the postoperative checkup, etc.

For ambulatory surgery, the patients should be accompanied by a responsible adult for $24 \mathrm{~h}$. The ambulatory patient should not drive his/her car $24 \mathrm{~h}$ postoperatively. There is no evidence regarding the timing of preoperative evaluation on patient outcome. Sufficient time before the planned intervention should allow additional tests (according to local resources and policy) or implementation of preoperative interventions aimed at improving patient outcome. Smoking cessation has shown to be beneficial at 4 to 8 weeks before the surgical procedure. Alcohol abstinence longer than 1 month has positive effects.

All patients should be well informed about the type of surgery and its most common complications and patients should give consent. A true informed consent is based on the knowledge of all the reasonably alternative options, the risks and benefits of each option, and the likelihood that these may occur [8]. In current practice, however, many clinicians, after a very brief oral explanation, request a patient's signature for a specific intervention, and consequently, given the patient-clinician trust relationship, the patient signs. However, since most interventions are elective, assessing the risk-benefit trade-offs is best done in a shared decision-making model. When the patient has a right of say in the decision making, the intervention is planned in true copartnership and the patient will be more understanding in the event of a complication. Of course, this process is only really valuable when there is no distinctive "best" treatment option. Nevertheless, even if there's only one surgical solution, the option of not performing the surgery can be discussed and the patient will at least have the feeling that his opinion is valued.

It is obvious that the complication rate varies according to the pathology, especially if the pathology gives rise to more invasive surgery. A surgical intervention, such as a hysterectomy for an advanced cancer, for example, is more prone to complications than a hysterectomy performed to treat benign gynecological pathology. Postoperative care should therefore be adapted not only to the type of intervention but also to the pathology: in oncological patients, the risk of thromboembolic disease, for example, is much higher and optimal thromboprophylaxis is mandatory.

\section{Material}

Surgery nowadays is getting more and more high-tech, implying the use of different energy sources, disposable devices, implantation materials, etc. For surgeons, nurses, and logistic personnel, good knowledge of the material used is essential. Especially in endoscopic surgery, whether it's robotically assisted or not, the technicality is becoming enormous, and endoscopic surgeons nowadays are expected to be genuine technical wizards.

When introducing a new technique and/or material, optimal training should be offered by the manufacturer; moreover, this training should be repeated with regular intervals, especially when new staff is involved. All material used has to be in optimal condition and should only be used for the designed purpose. A hospital or department should consider standardizing equipment as much as possible.

The hospital should have a quality control system to check the operative equipment with regular intervals and replace or repair it when necessary. Complications, such as burns, infections, or left parts of devices in the patient's body, can be due to deficient material. For all routine interventions, checklists have to be available summing up all the necessary material. Before the intervention, all material should be checked on its integrity and function by the operating nurse and should be double checked by the surgeon before use. At the end of the intervention, the surgical material and gauzes should be counted and this is noted in the operative report. The surgical specimen should be correctly labelled and sent to the pathology lab. All unrelated distractions (magazines, mobile phones, etc.) should be removed from the operating theater.

All medication used before, during, and after surgery has to be administered on instruction of the medical doctor and should be noted in the patient's file. Also patient's home medication should be carefully noted and continued if appropriate. When a potentially hazardous product is administered, the correctness of this application should be checked by two people. When the patient is discharged, clear written information has to be provided on the dose and frequency of newly started medication.

\section{Procedure}

Sometimes the same pathology can be treated through different interventions: for a woman with bleeding disorders, a hysterectomy can be performed abdominally, vaginally, or laparoscopically. Moreover, a total or subtotal hysterectomy can be chosen; a less invasive procedure, such as an endometrial ablation, can also be considered. The complication rates can vary according to the route or invasiveness of the intervention. If different procedures exist for one and the same pathology, these different options should be discussed with the patient, including differences in complication rates, recovery, recurrence, absence from work, etc.

\section{Quality assurance and assessment tools currently used}

Large (national) databases

In the USA as well as in several Nordic countries, large national databases were established to record surgical 
outcome. The National Surgical Quality Improvement Program (NSQIP) in the USA was established in the early nineties of the previous century to record risk-adjusted surgical morbidity, rate hospital quality, and benchmark surgical performance [9]. These databases have the potential of revealing specific high-risk groups per intervention and could have an influence on decision making. Furthermore, they could give a load of information on the origin of complications. However, such initiatives were slowed down by the changing medicolegal climate (lawsuits) and the enormous costs of data collection. Instead, administrative databases are being increasingly used; however, these are associated with significant lower reliability [10]. Clinical databases, on the other hand (made and controlled by clinicians), might underreport complications [11]; nevertheless, such databases are frequently used for benchmarking between hospitals. However, data recorded in such databases is mostly gathered by residents and trainees, and recent research has showed that outcome assessment by residents is quite unreliable [12]. Therefore self-reported quality reports are likewise largely inaccurate. Moreover, most databases do not record the comorbidity, thus making the outcome hard to compare. Finally, local and national complication registries try to gather information on the encountered complications. However, the weakness of a complication registry is the fact that it only serves when there is a complication, and at that moment, it is easily "forgotten." Postoperative complications are equally underreported, especially if these occur after the patient has been discharged.

\section{Surgical checklists}

Checklists are since long widely used in all kinds of professional sectors; in hospitals, however, they have only been introduced recently. In 2009, a study conducted by the "Safe Surgery Safes Lives" study group, using a 19-item surgical safety checklist, was published in the New England Journal of Medicine. This study showed that the use of the checklist improved safety and quality of surgery around the world (both in industrialized as in low-resource countries) [13]. The rate of death declined from $1.5 \%$ before the checklist was introduced to $0.8 \%$ afterwards $(P=0.003)$. Inpatient complications occurred in $11 \%$ of patients at baseline and fell to $7 \%$ after introduction of the checklist $(P<0.001)$.

An important factor is the introduction of a "surgical time out:" a moment of reflection before incision where all members of the team (nurses, surgeons, anesthesiologists, etc.) confirm that they all are aware of each other's functions and responsibilities, the patient's identity, the pathology and intervention, the surgical site, the anticipated blood loss, the necessity of antibiotics, etc. Only when all of these factors have been checked, the intervention is allowed to commence.

\section{Process analysis}

The risk of complications increases when a novel technique or approach is introduced without first evaluating the care team's comfort with the change, the team's recognition of potential problems and the team member's understanding of each of their roles. Only rarely proactive steps are taken to identify hazards and design systems to minimize the risk of complications and improves patient outcome. One such program is an initiative for proactive risk analysis by the Department of Veteran Affairs, which uses failure mode and effects analysis (FMEA). The FMEA is a proactive error prevention system designed to identify problems in systems before any adverse events occur. This methodology has been used successfully for reducing errors in medication administration, blood transfusion, and clinical laboratories [14]. However, the application of FMEA in health care delivery is limited.

Important information can be revealed by conducting in situ simulation, i.e., in the same location where actual care takes place, using the same resources and involving actual health-care team members and existing processes (a kind of "mock-procedure").

Even for processes which are already in use a thorough analysis can reveal possible limitations or hazards. Therefore, quality assurance (QA) programs are implemented to monitor and evaluate efficiency and standards of care. In an Australian study, medical students were asked to follow a patient from admission to discharge. Afterwards, these students were very well able to identify QA issues and even to propose solutions [15]. Categories of problems highlighted by these students included inappropriate patient and procedure selection, inadequate pain management, discharge, communication and resource issues. Students made a number of recommendations and they also developed new guidelines and protocols.

\section{Surgical simulation training}

Surgical simulation training (both procedural training and surgical team training) are rapidly becoming necessary adjuncts to traditional patient-based training models. Such training provides a safe and ethical acceptable way to acquire the necessary surgical skills before entering an operative theater. Skills acquired through such simulation training seem to be very well transferable to the operative setting. [16]. A systematic review by Cook et al. [17] in the JAMA of 2011 showed that, for health professions education, technology-enhanced simulation training (both computer-assisted and not), in comparison with no intervention, is consistently associated with large effects for the outcomes of knowledge, time skills, process skills, and behaviors. 
Key performance indicators

Key performance indicators (KPI) are quantifiable measurements, agreed to beforehand, that reflect the critical success factors of an organization. They can also be used for measuring the success of medical interventions such as surgery. The readmission rate, for example, can be used as a KPI to measure the complication rate of a certain department or even a specific type of intervention. KPIs can also be used to benchmark between different institutions.

In France, every surgeon is expected to register every serious adverse event in a national registry. Analysis of this data showed that very different dynamics occurred, which justifies systematic analysis of different serious events by their nature or context of occurrence. (http://www.drees.sante.gouv.fr).

\section{Future perspectives}

Create one comprehensive medical file per patient and promote the use of it

During the course of his life, each patient will be treated by more than a dozen of physicians on average. In general, each of these physicians will keep his own medical file, and most of these medical files will lack important information on the patient's history. It seems obvious that, as each patient nowadays has an electronic identity (e-ID), the creation of an electronic medical file (e-MF) would have a beneficial effect on the communication between physicians and eventually on the safety of our medical actions. Clinicians would have to access the patient's medical file upon each contact (ambulatory or hospitalization) and fill-in the reasons for encounter, the probable diagnosis and prescribed therapy. This way late onset postoperative complications will not be missed.

Organize nationwide prospective registration of all surgical interventions

The weakness of a complication registry lies in the fact that it only has to be accessed when a complication occurs, and at that moment, it is easily "forgotten." Therefore, all surgical interventions should be entered into a prospective national registry. A surgical episode should start at the moment that the surgery is planned and should only be closed after a fixed postoperative period (e.g., 6 weeks for a cholecystectomy, 12 weeks after an extra-uterine pregnancy, etc.).

Offer postgraduate training for surgeons

In 2004, the Flemish Society of Obstetrics and Gynecology (VVOG) performed an inquiry into the needs for postoperative training among Flemish gynecologists. From this inquiry, it became clear that more than $80 \%$ of respondents desired specific endoscopic workshops adapted to their needs. Since then, the Special Interest Group on Gynecological Endoscopy started organizing laparoscopic workshops according to a three level system:

- A first level includes stereotactic exercises and suturing/ knotting exercises (dry-lab) plus a simple exercise on tissue handling on a rabbit model.

- A second level includes more suturing and knotting exercises (different types, equally dry-lab), several presentations on the theory plus the practical implementation (video demonstrations) of some more advanced laparoscopic interventions (hysterectomy, myomectomy, and deep infiltrating endometriosis) plus a full-day hands-on training in a pig's lab.

- A third level aims at training the technique of laparoscopic hysterectomy and the dissection of the pelvic sidewall on female Thiel-embalmed corpses $[18,19]$.

Participants are only allowed to enter a higher level after accomplishing the level below.

Introduce an accreditation system for surgeons for specific interventions

This is a natural consequence of all of the above: better-trained surgeons will yield less complications and will lead to a cost reduction of our health system. Nowadays, every surgeon is allowed to start using a new technique whenever he/she feels up to it. Up to now, a new surgical technique is largely mastered through apprenticeship and self-study (results from a survey by the Flemish Society of Obstetrics and Gynecology in 2010). However, the introduction of the European Working Time Directive and the pressure to increase surgical productivity have reduced the possibility to learn surgical skills in the operating theater [20]. Moreover, surgery is becoming more and more complex, and a basic specialist training is by far not sufficient to master all interventions. Moreover, basis surgical skills (stereotaxis, knotting skills, etc.) should ideally be acquired before entering the operating theater and performing surgery on patients. Since many years in the UK, there has been an ongoing debate about surgical competence. The majority view is that surgical competence should be based on clinical judgement, operative skills, and cognitive ability. The assessment of technical ability should be based on standardized checklists. Also, the call for competence checks during the professional career of surgeons (e.g., every 5 years) becomes louder [20, 21].

An accreditation system for at least some interventions seems necessary. To get accreditation, surgeons should follow a specific training to make sure that they have the necessary material and perform a minimal number of interventions. 
Accredited surgeons will eventually have less complications; moreover, the unnecessary application of some procedures will decrease. The European Society of Gynecological Endoscopy (ESGE), together with the European Academy of Gynecological Surgery, has recently initiated such an accreditation system. This so-called Gynecological Endoscopic Surgical Education and Assessment (GESEA) program is made up of three levels and aims at giving gynecologists a comprehensive endoscopic training based on five pillars of competence: elearning and self-assessment, training of specific endoscopic skills on pelvic trainers, surgical knowledge assessment (by means of multiple choice tests), surgical practice curriculum (document endoscopic interventions), and continuing medical education (attendance at congresses and workshops; http:// www.esge.org/education/guidelines).

\section{Conclusions}

Several important factors can be identified which are influencing the quality of surgical care. These factors can be classified into four major groups: the team of caretakers, the patient, the material, and the procedure. For all of these factors, a high level of knowledge and optimal communication is crucial to guarantee a high standard of care and minimize the chance of complications.

Databases of surgical procedures have the potential to offer an enormous amount of information on the quality of care. However, the implementation of comprehensive databases is difficult and expensive, while its value is overshadowed by possible underreporting.

Introducing surgical checklists is a cheap yet efficient way to increase both the safety and the quality of surgical care not only on a local level but also worldwide. Nevertheless, its implementation is sometimes opposed since they slow down the patient flow. However, this so called surgical time-out is in fact its most important mode of action.

The risk of complications tends to increase when a new technique is introduced. But even for processes which are already in use, a thorough analysis can reveal possible limitations or hazards. Therefore, quality assurance programs have to be implemented. Also surgical simulation training is rapidly becoming a necessary adjunct to traditional patient-based training models. Finally, key performance indicators can be used for measuring the success of medical interventions such as surgery.

For the near future, the introduction of one comprehensive medical file per patient could be a major step in optimizing the communication between caretakers and increasing the safety and efficiency of our medical deeds. In parallel, a nationwide prospective registry for surgical interventions should be introduced. The physician's fee could be made partially dependent on the use of the above tools.
As compared to a few decades ago, new surgical techniques are far more rapidly introduced and adopted by surgeons. Therefore, a newly trained specialist can never master all interventions of its discipline, and postgraduate training is becoming more and more important. Such postgraduate training can very well be organized by the national professional groups and should be adapted to the local needs. A system of accreditation for specific interventions should be introduced guaranteeing their state-of-the-art application. In the hands of well-trained surgeons, these specific interventions will generate less complications and will be restrained to its correct applications.

Author's roles All the authors were involved in drafting and revision of article and final approval of submitted manuscript.

Funding No external funding was sought for this study.

Conflict of interest None of the authors has any conflicts of interest to declare.

\section{References}

1. Dindo D, Clavien PA (2010) Quality assessment in surgery: mission impossible? Patient Saf Surg 4:18

2. Arora S, Sevdalis N, Nestel D, Woloshynowych M, Darzi A, Kneebone R (2010) The impact of stress on surgical performance: a systematic review of the literature. Surgery 147:318-330

3. Wolf FA, Way LW, Stewart L (2010) The efficacy of medical team training: improved team performance and decreased operating room delays: a detailed analysis of 4863 cases. Ann Surg 252:477-485

4. Neily J, Mills PD, Young-Xu Y et al (2010) Association between implementation of a medical team training program and surgical mortality. JAMA 304:1693-1700

5. De Hert S, Imberger G, Carlisle J et al (2011) Preoperative evaluation of the adult patient undergoing non-cardiac surgery: guidelines from the European Society of Anaesthesiology. Eur J Anaesthesiol 28: 684-722

6. Smith TB, Stonell C, Pukayastha S et al (2009) Cardiopulmonary exercise testing as a risk assessment method in non cardiopulmonary surgery: a systematic review. Anaesthesia 64:883-893

7. Murthy BV (2006) Improving the patient's journey. The role of the preoperative assessment team. R Coll Anaesthesist Bull 37:1885-1887

8. Weinstein JN, Clay K, Morgan TS (2007) Informed patient choice: patient-centred valuing of surgical risks and benefits. Health Aff 26: 726-730

9. Rowell KS, Turrentine FE, Hutter MM, Khuri SF, Henderson WG (2007) Use of national surgical quality improvement program data as a catalyst for quality improvement. J Am Coll Surg 204:1293-1300

10. Best WR, Khuri SF, Phelan M et al (2002) Identifying patient preoperative risk factors and postoperative adverse events in administrative databases: results from the Department of Veterans Affairs national surgical quality improvement program. J Am Coll Surg 194:257-266

11. Gunarsson U, Seligsohn E, Jestin P et al (2003) Registration and validity of surgical complications in colorectal cancer surgery. Br J Surg 90:454-459

12. Dindo D, Hahnloser D, Clavien PA (2010) Quality assessment in surgery—riding a lame horse. Ann Surg 251:766-771 
13. Haynes AB, Weiser TG, Berry WR et al (2009) A surgical safety checklist to reduce morbidity and mortality in a global population. NEJM 360:491-499

14. Rodriguez-Paz JM, Mark LJ, Herzer KR et al (2009) A novel process for introducing a new intra-operative program: a multidisciplinary paradigm for mitigating hazards and improving patient safety. Anesth Analg 108:202-210

15. Rudkin GE, O’Driscoll MC, Limb R (1999) Can medical students contribute to quality assurance programmes in day surgery? Med Educ 33:509-514

16. Sturm LP, Windsor JH, Cosman PH et al (2008) A systematic review of skills transfer after surgical simulation training. Ann Surg 248: 166-179
17. Cook DA, Hatala R, Brydges R et al (2011) Technology-enhanced simulation for health professions education. A systematic review and meta-analysis. JAMA 306:978-988

18. Thiel W (1992) The preservation of the whole corpse with natural color. Ann Anat 174:185-195

19. Tjalma WA, Degueldre M, Van Herendael B, D’Herde K, Weyers S (2013) Postgraduate cadaver surgery: an educational course which aims at improving surgical skills. Fact Views Vis Obgyn 5:61-65

20. Moorthy K, Munz Y, Sarker SK, Darzi A (2003) Objective assessment of technical skills in surgery. BMJ 327:1032-1037

21. Cuschieri A, Francis N, Crosby J, Hanna GB (2001) What do master surgeons think of surgical competence and revalidation? Am J Surg $182: 110-116$ 